\title{
Nonlinear optimization for human-like synchronous movements of a dual arm-hand robotic system
}

\author{
G. Gulletta*, S. M. Araújo*, E. Costa e Silva ${ }^{\dagger}$, M. F. Costa**, W. Erlhagen** \\ and E. Bicho* \\ *Centre Algoritmi/Dept. of Industrial Electronics, University of Minho, Portugal \\ ${ }^{+}$CIICESI, ESTGF, Polytechnic Institute of Porto, Apt. 205, Felgueiras, Portugal \\ ${ }^{*}$ Centre for Mathematics/Dept. of Mathematics and Applications, University of Minho, Portugal
}

\begin{abstract}
In previous work we have presented a model capable of generating human-like movements for a dual arm-hand robot involved in human-robot cooperative tasks. However, the focus was on the generation of reachto-grasp and reach-to-regrasp bimanual movements and no synchrony in timing was taken into account. In this paper we extend the previous model in order to accomplish bimanual manipulation tasks by synchronously moving both arms and hands of an anthropomorphic robotic system. Specifically, the new extended model has been designed for two different tasks with different degrees of difficulty. Numerical results were obtained by the implementation of the IPOPT solver embedded in our MATLAB simulator.
\end{abstract}

Keywords: large-scale nonlinear optimization problems, bimanual human-like movements, synchronous movements, dual arm-hand anthropomorphic robot, IPOPT

PACS: $02.60 . \mathrm{Pn}$

\section{INTRODUCTION}

In order to implement human-like synchronous movements in a bimanual anthropomorphic robot, it is necessary to understand the basis of bimanual coordination in humans and the functional models (based on neuro-physiological evidences) that have been proposed.

Recent data acquired address three conceptual areas [1]: the generalized motor program (GMP), intermanual crosstalk, dynamic system models. Although controversial studies exist on each one of these approaches, the proposed motion planner takes inspiration by those models that represent synergies of muscles easily controlled jointly as a singular functional unit and explain the strong tendency for syn-

Task 1
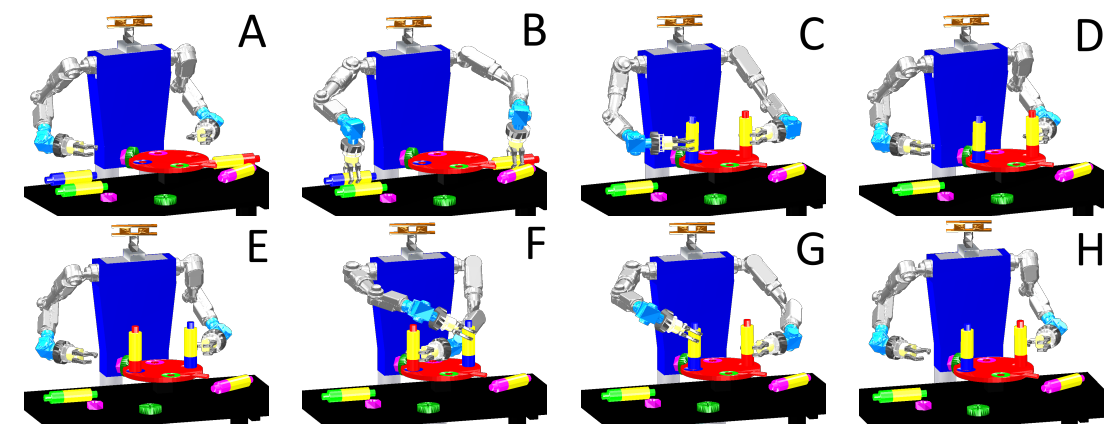

Figure 1. ARoS is an anthropomorphic robot equipped with two 7 degrees of freedom (DOFs) arms and two 4 DOFs hands. Panels A-D and panels E-H show screenshots obtained in our MATLAB simulator related to the execution of Task 1 and Task 2 respectively. In Task 1 the robot has to reach and grasp the two columns (B) starting from its initial posture (A). Next, ARoS has to insert the columns in the right holes into the base (C) and, finally, it goes back to its initial posture (D). In Task 2 the robot has to reach and grasp the two columns by crossing its arms (F), starting from its initial posture (E). Then, ARoS has to swap the columns to insert them in the right hole into the base (G). Finally, the robot return to its initial posture $(\mathrm{H})$. 
chronous timing of bimanual movements (e.g. [2, 3, 4]). Specifically, we designed a planner for generating complex sequences of synchronous bimanual movements that are performed in scenario cluttered with obstacles (e. g. the body of the robot, the table and others objects as shown in Figure 1). Even though in the last decade several synchronous bimanual motion planning techniques have been presented (e.g. [5, 6]), poor attention has been paid to the human-likeness and to cognitive effects in motion planning. Strongly inspired on the Posture-Based Motion Planning Model $([7,8])$ of humans upper-limbs movements, our motion planner [9] has been extended to generate human-like bimanual synchronous movements which explicitly reveal their underlying intention to obtain a natural and efficient human-robot interaction in cooperative tasks.

\section{BIMANUAL SYNCHRONOUS MOVEMENT PLANNING AS A NONLINEAR CONSTRAINED OPTIMIZATION PROBLEM}

The formalization of the motion planner is presented in this section (for further details see [9]). The configuration of the arms-hands in the joint space is represented by the 22-dimensional vector $\boldsymbol{\theta}=$ $\left(\boldsymbol{\theta}_{1}, \boldsymbol{\theta}_{2}, \ldots, \boldsymbol{\theta}_{22}\right)^{T}$. $\boldsymbol{\theta}_{R}=\left(\boldsymbol{\theta}_{1}, \ldots, \boldsymbol{\theta}_{11}\right)^{T}$ refers to the right arm-hand, while $\boldsymbol{\theta}_{L}=\left(\boldsymbol{\theta}_{12}, \ldots, \boldsymbol{\theta}_{22}\right)^{T}$ refers to the left arm-hand. The trajectory of the joints is formulated as follows:

$$
\begin{aligned}
\boldsymbol{\theta}\left(t, \boldsymbol{\theta}_{f}, \boldsymbol{\theta}_{b}\right)=\boldsymbol{\theta}_{0} & +\left(\boldsymbol{\theta}_{f}-\boldsymbol{\theta}_{0}\right)\left(10 \tau^{3}-15 \tau^{4}+6 \tau^{5}\right) \\
& +\boldsymbol{\omega}_{0} T\left(\tau-6 \tau^{3}+8 \tau^{4}-3 \tau^{5}\right)+\boldsymbol{\omega}_{f} T\left(-4 \tau^{3}+7 \tau^{4}-3 \tau^{5}\right) \\
& +\frac{1}{2} \boldsymbol{\alpha}_{0} T^{2}\left(\tau^{2}-3 \tau^{3}+3 \tau^{4}-\tau^{5}\right)+\frac{1}{2} \boldsymbol{\alpha}_{f} T^{2}\left(\tau^{3}-2 \tau^{4}+\tau^{5}\right) \\
& +\left(\boldsymbol{\theta}_{b}-\boldsymbol{\theta}_{0}\right) \sin ^{2}\left(\pi \tau^{\vartheta}\right)
\end{aligned}
$$

where the parameters $\boldsymbol{\theta}_{0}, \omega_{0}, \boldsymbol{\alpha}_{0} \in \mathbb{R}^{n j}$ are constant vectors that represent initial joints position, velocity and acceleration of the two arms-hands, respectively. While parameters, $\boldsymbol{\theta}_{f}, \boldsymbol{\omega}_{f}, \boldsymbol{\alpha}_{f} \in \mathbb{R}^{n j}$ represent position, velocity and final joints acceleration of the two arms-hands, respectively. $T \in \mathbb{R}^{+}$represents the movement duration, $t \in[0, T], \tau=\frac{t}{T} \in[0,1]$ is the normalized movement duration and $\vartheta=-\frac{\ln _{2}}{\ln _{t_{b}}}$, where $\left.t_{b} \in\right] 0,1[$ that is the parameter to control when the bounce posture is reached. Therefore, $t \in[0, T]$ is divided in $N_{T}$ equally spaced steps $t_{i}=i \Delta$, where $\Delta=\frac{T}{N_{T}}$ is the step size and $i=0,1, \ldots, N_{T}$. Consequently, $\boldsymbol{\theta}\left(t_{i}, \boldsymbol{\theta}_{f}, \boldsymbol{\theta}_{b}\right)$ represents the composite trajectory at time $t_{i}$. The number of joints, $n_{j}$, depends on the type of the movement to plan (see Tables 2 and 3). The calculus of a composite trajectory is posterior to the selection of a goal posture, $\boldsymbol{\theta}_{f}$, and of the bounce posture, $\boldsymbol{\theta}_{b}$. This selection process is divided into two sub-problems: goal posture selection $(\mathbf{P a})$ and a bounce posture selection $(\mathbf{P b})$. These two sub-problems are modeled as nonlinear constrained optimization problems:

$$
\text { (Pa) } \begin{aligned}
\min _{\boldsymbol{\theta}_{f} \in \mathbb{R}^{14}} & \sum_{k=1}^{14} \lambda_{k}\left(\theta_{0, k}-\theta_{f, k}\right)^{2}, \lambda_{k} \geq 0 \\
\text { s.t. } & \boldsymbol{c}_{1 A}\left(\boldsymbol{\theta}_{f A}\right)=\mathbf{0} \\
& \boldsymbol{c}_{2 A}\left(\boldsymbol{\theta}_{f A}\right)=\mathbf{0} \\
& \overline{\boldsymbol{h}}_{f A}\left(\boldsymbol{\theta}_{f A}, \zeta_{\text {tar }}\right) \geq \mathbf{0} \\
& \underline{\boldsymbol{h}}_{f A}\left(\boldsymbol{\theta}_{f A}, \zeta_{o b s}\right) \geq \mathbf{0} \\
& \boldsymbol{q}_{f}\left(\boldsymbol{\theta}_{f L}, \boldsymbol{\theta}_{f R}, \gamma\right) \geq \mathbf{0} \\
& \boldsymbol{\theta}_{m A} \leq \boldsymbol{\theta}_{f A} \leq \boldsymbol{\theta}_{M A}
\end{aligned}
$$

(Pb) $\min _{\boldsymbol{\theta}_{f} \in \mathbb{R}^{n_{j}}} \sum_{k=1}^{n_{j}} \lambda_{k}\left(\theta_{0, k}-\theta_{b, k}\right)^{2}, \lambda_{k} \geq 0$

$$
\begin{array}{ll}
\text { s.t. } & \boldsymbol{\theta}_{m A} \leq \boldsymbol{\theta}_{A}\left(t_{i}, \boldsymbol{\theta}_{f A}, \boldsymbol{\theta}_{b A}\right) \leq \boldsymbol{\theta}_{M A} \\
& \overline{\boldsymbol{h}}_{b A}\left(\boldsymbol{\theta}_{A}\left(t_{i}, \boldsymbol{\theta}_{f A}, \boldsymbol{\theta}_{b A}\right), \varepsilon_{t a r}\left(t_{i}\right)\right) \geq \mathbf{0}
\end{array}
$$$$
\underline{\boldsymbol{h}}_{b A}\left(\boldsymbol{\theta}_{A}\left(t_{i}, \boldsymbol{\theta}_{f A}, \boldsymbol{\theta}_{b A}\right), \varepsilon_{o b s}\left(t_{i}\right)\right) \geq \mathbf{0}
$$$$
\boldsymbol{q}_{b}\left(\boldsymbol{\theta}_{L}\left(t_{i}, \boldsymbol{\theta}_{f L}, \boldsymbol{\theta}_{b L}\right), \boldsymbol{\theta}_{R}\left(t_{i}, \boldsymbol{\theta}_{f R}, \boldsymbol{\theta}_{b R}\right), \psi\left(t_{i}\right)\right) \geq \mathbf{0}
$$$$
\boldsymbol{\theta}_{m A} \leq \boldsymbol{\theta}_{b A} \leq \boldsymbol{\theta}_{M A}
$$$$
, t_{i}=0, \ldots, T
$$

where the constant vectors $\boldsymbol{\theta}_{m A}$ and $\boldsymbol{\theta}_{M A}$ represent the lower and upper joint limits of each arm $A \in\{R, L\}$

respectively . $\gamma>0$ is the clearance distance between the arms-hands at the final posture, while $\zeta_{\text {tar }}>0$ and $\zeta_{\text {obs }}>0$ represent the clearance distance between one arm-hand and the target of the other hand and 
between one arm-hand and obstacles, respectively at the final posture. Equations (3), (4) are nonlinear constraints of the target position and orientation the position and the orientation of the arm and hand, respectively obtained using direct kinematics (for details see [9]). $\psi\left(t_{i}\right)>0$ is the clearance distance between the arms-hands at time $t_{i}$, while $\varepsilon_{\text {tar }}\left(t_{i}\right)>0$ and $\varepsilon_{o b s}\left(t_{i}\right)>0$ are functions of time representing the clearance distance between one arm-hand and the target of the other arm-hand and between one arm-hand and obstacles at time $t_{i}$, respectively. Inequalities (5) and (11) are nonlinear constraints of one arm-hand in relation with the target position of the other arm-hand. Similarly, inequalities (6) and (12) are nonlinear constraints of one arm-hand and the obstacles. For further details on constraints formulation see [9]. Finally, inequalities (7) and (13) are non-linear constraints for avoiding collisions between the arms-hands .

\section{RESULTS}

We focus on the sequence of movements in the two different tasks and presented in Figure 1 and described in Table 1. The numerical results were obtained using an Intel(R) Core(TM) i7-4770 CPU@ 3.40GHz running Windows 8 64-bits with a AMD Radeon HD 6570 video card and 8GB of RAM Memory. All the optimization problems in Table $1(\mathrm{P \# a}$ and $\mathrm{P \# b}$ ) were coded in AMPL modeling language and solved using IPOPT 3.11 [10]. The solver is provided by OPTI Toolbox [11]. During the movements, all joints move synchronously and they start and end each movement in static conditions, i.e. in (1) $\boldsymbol{\omega}_{0}=\mathbf{0}, \boldsymbol{\omega}_{f}=\mathbf{0}$, $\boldsymbol{\alpha}_{0}=\mathbf{0}$ and $\boldsymbol{\alpha}_{f}=\mathbf{0}$. In practice all equality constraints were transformed into inequalities considering its squared euclidean norm and using constants $\beta>0$, in (3) and $\delta>0$ in (4).

The number of P\#a problems for each movement is arbitrary and it is usually chosen according the difficulty of the motion. For example, swapping the columns in Task $\mathbf{2}$ is a highly risky movement, therefore we preferred to execute it in three steps. The dimension of the P\#a problems depends on the number of constraints in (5) and in (6). In Task 1 the constraints (7) and (13) were not considered because the arms-hands do not share the same workspace during the movements.

Table 1. Tasks formulation

\begin{tabular}{cccccccccccc}
\hline Task & Movement & $\beta$ & $\delta$ & $\zeta_{\text {tar }}$ & $\zeta_{\text {obs }}$ & $\gamma$ & $\lambda_{k}$ & $\mathbf{T}$ & $\boldsymbol{t}_{\boldsymbol{b}}$ & $\begin{array}{l}\text { Final Posture } \\
\text { Selection }\end{array}$ & $\begin{array}{c}\text { Bounce Posture } \\
\text { Selection }\end{array}$ \\
\hline \multirow{3}{*}{1} & $\begin{array}{l}\text { Reach to } \\
\text { grasp }\end{array}$ & 1 & $10^{-5}$ & 10 & 5 & 1 & 1 & 1 & 0.5 & P1a1 + P1a2 & P1b \\
& $\begin{array}{l}\text { Transport } \\
\text { to insert }\end{array}$ & 1 & $10^{-5}$ & 10 & 5 & 1 & 1 & 1 & 0.5 & P2a1 + P2a2 & P2b \\
\hline $\begin{array}{l}\text { Return } \\
\text { to home }\end{array}$ & 1 & $10^{-5}$ & 10 & 5 & 1 & 1 & 1 & 0.5 & P3a1 & P3b \\
\hline $\begin{array}{l}\text { Reach to } \\
\text { grasp }\end{array}$ & 1 & $10^{-4}$ & 10 & 5 & 1 & 1 & 1 & 0.5 & P1a1 + P1a2 & P1b \\
\hline $\begin{array}{l}\text { Swap to } \\
\text { insert }\end{array}$ & 1 & $10^{-5}$ & 10 & 5 & 1 & 1 & 1 & 0.5 & P2a1 + P2a2 + P2a3 & P2b \\
\hline $\begin{array}{l}\text { Return } \\
\text { to home }\end{array}$ & 1 & $10^{-3}$ & 10 & 5 & 1 & 1 & 1 & 0.5 & P3a1 & P3b \\
\hline
\end{tabular}

Tables 2 and 3 provide results regarding the optimal solution found by the solver for the final posture and the bounce posture selection problems for all movements in Task $\mathbf{1}$ and Task $\mathbf{2}$. Here $N$ is the number of variables and it corresponds to $n_{j}$ in $\mathbf{P} \# \mathbf{b}$ problems. Moreover, $M$ is the number of inequality constraints, Obj is the objective function value and CPU is the computational time in seconds.

For all P\#a problems, IPOPT was able to find an optimal solution in less then 0.9 seconds. The dimension of the $\mathbf{P} \# \mathbf{b}$ problems was dependent on the number of constraints in (11) and in (12) . For all P\#b problems, IPOPT was able to find a solution in less than 2.5 seconds. P2b was the most risky movement and IPOPT could find a solution only after 2.34 seconds. However, P1a1 for both tasks were featured by the highest direct component as the value of the objective function shows. This result is due to the fact that both arms need to perform large movements (very distant from their initial posture) to reach and grasp their targets. 
Table 2. Task 1: numerical results for P\#a and P\#b problems

\begin{tabular}{c|cc|c||cc|c||cc}
\hline & P1a1 & P1a2 & P1b & P2a1 & P2a2 & P2b & P3a1 & P3b \\
\hline $\mathbf{N}$ & 14 & 14 & 18 & 14 & 14 & 14 & 14 & 18 \\
$\boldsymbol{N}_{\boldsymbol{t}}$ & - & - & 15 & - & - & 20 & - & 10 \\
$\boldsymbol{M}$ & 226 & 226 & 2406 & 226 & 226 & 1984 & 272 & 1531 \\
Obj & 17.997 & 0.61 & 0.085 & 4.106 & 3.362 & 0.021 & 0.077 & 0.034 \\
$\mathbf{C P U}$ & 0.87 & 0.43 & 1.01 & 0.63 & 0.41 & 1.14 & 0.62 & 1.53 \\
\hline
\end{tabular}

Table 3. Task 2: numerical results for P\#a and P\#b problems

\begin{tabular}{c|cc|c||ccc|c||cc}
\hline & P1a1 & P1a2 & P1b & P2a1 & P2a2 & P2a3 & P2b & P3a1 & P3b \\
\hline $\mathbf{N}$ & 14 & 14 & 18 & 14 & 14 & 14 & 14 & 14 & 18 \\
$\boldsymbol{N}_{\boldsymbol{t}}$ & - & - & 20 & - & - & - & 10 & - & 10 \\
$\boldsymbol{M}$ & 151 & 151 & 1597 & 151 & 151 & 116 & 1792 & 116 & 566 \\
Obj & 15.886 & 0.102 & 0.137 & 0.102 & 1.40 & 0.124 & 0.033 & 0.421 & 0.196 \\
$\mathbf{C P U}$ & 0.33 & 0.40 & 2.17 & 0.56 & 0.31 & 0.22 & 2.34 & 0.56 & 0.67 \\
\hline
\end{tabular}

\section{CONCLUSIONS AND FUTURE WORK}

In this paper we have presented a formulation of a motion planner capable of generating bimanual synchronous movements for an anthropomorphic robot system. These movements are smooth and exhibit features (e.g. hand velocity profile, synchrony in timing) observed in human upper-limb movements in performing similar tasks. The results suggest that the planning process is sufficiently fast to guarantee human-robot interaction and for performing an eventual re-planning of the trajectories in case of changes in the scenario during the execution of the movement. In the short term an integration of an efficient technique to cope with changes in the scenario is a priority in our work.

\section{ACKNOWLEDGMENTS}

This work was financed by EU funded Project PF7 Marie Curie “NETT - Neural Engineering Transformative Technologies".

\section{REFERENCES}

1. S. Cardoso de Oliveira, Acta Psychol. (Amst). 110, 139-59 (2002), ISSN 0001-6918.

2. K. Shockley, D. C. Richardson, and R. Dale, Top. Cogn. Sci. 1, 305-319 (2009), ISSN 17568757.

3. C. M. L. Hughes, P. Reissig, and C. Seegelke, Acta Psychol. (Amst). 138, 111-8 (2011), ISSN 1873-6297.

4. G. P. Bingham, K. Hughes, and M. Mon-Williams, Exp. brain Res. 184, 283-93 (2008), ISSN 1432-1106.

5. N. Vahrenkamp, M. Do, T. Asfour, and R. Dillmann, 2010 IEEE Int. Conf. Robot. Autom. pp. $2883-2888$ (2010).

6. F. Zacharias, C. Schlette, F. Schmidt, C. Borst, J. Rossmann, and G. Hirzinger, 2011 IEEE Int. Conf. Robot. Autom. pp. 1192-1198 (2011).

7. D. A. Rosenbaum, R. J. Meulenbroek, J. Vaughan, and C. Jansen, Psychol. Rev. 108, 709-734 (2001).

8. J. Vaughan, and D. A. Rosenbaum, "Modeling Reaching and Manipulating in 2- and 3-D Workspaces : The Posture-Based Model," in Int. Conf. Dev. Learn., 2006, vol. 01, pp. 3-8.

9. E. Costa e Silva, M. F. Costa, E. Bicho, and W. Erlhagen, "Nonlinear Optimization for Human-Like Movements of a High Degree of Freedom Robotics Arm-Hand System," in ICCSA, edited by B. Murgante, Springer-Verlag, 2011, vol. 6784, pp. 327-342.

10. A. Wächter, and L. T. Bielger, Math. Program. pp. 1-33 (2005).

11. J. Currie, and D. I. Wilson, "OPTI: Lowering the Barrier Between Open Source Optimizers and the Industrial MATLAB User," in Foundations of Computer-Aided Process Operations, edited by N. Sahinidis, and J. Pinto, Savannah, Georgia, USA, 2012. 